\title{
Potential of DNA zygosity tests for non-invasive evaluation of risk of complications in twin pregnancies
}

\author{
Agnieszka Dziennik ${ }^{1}$, Krzysztof Preis ${ }^{2}$ (D), \\ Malgorzata Swiatkowska-Freund ${ }^{2} \mathbb{D}$, Krzysztof Rebala ${ }^{1} \mathbb{D}$ \\ ${ }^{1}$ Department of Forensic Medicine, Medical University of Gdansk, Poland \\ ${ }^{2}$ Department of Obstetrics, Medical University of Gdansk, Poland
}

\begin{abstract}
Objectives: To evaluate and compare the potential of DNA analysis and ultrasound examination for diagnosis of high-risk and low-risk twin pregnancies.

Material and methods: Chorionicity of 42 twin pregnancies was determined by routine high-resolution sonographic examination between 10 and 14 weeks of gestation. Zygosity was analysed in umbilical cord blood samples collected immediately after the birth by genotyping of 22 autosomal short tandem repeats used in human identity testing.

Results: Routine ultrasound imaging in the first trimester of twin gestations revealed 21 low-risk dichorionic (50\%) and 21 high-risk monochorionic pregnancies (50\%). DNA typing of umbilical cord blood showed 23 twin pairs with different genotypes (low-risk dizygotic pregnancies, 55\%) and 19 twin pairs with identical genotypes (high-risk monozygotic pregnancies, 45\%). We found four pregnancies (10\%), which were diagnosed sonographically as monochorionic diamniotic, but were identified as dizygotic in postnatal DNA testing. They constituted $19 \%$ of all high-risk monochorionic pregnancies detected by ultrasound imaging.

Conclusions: Our results indicate high potential of prenatal DNA testing of zygosity in identification of low-risk and high-risk twin gestations requiring different prenatal care, especially in cases when chorionicity and zygosity cannot be reliably determined by ultrasound examination and as a supplementary test able to detect gestations misdiagnosed as monochorionic, resulting from fusions of dizygotic placentas. In such cases, dizygosity detected prenatally eliminates the need for frequent prenatal visits typical for monochorionic pregnancies. If chorionicity cannot be unequivocally determined and a prenatal DNA test detects monozygotic twins, a more pessimistic variant of monochorionic pregnancy should always be assumed. Key words: twin pregnancy; prenatal diagnosis; ultrasonography; DNA testing; zygosity of twin pregnancy; chorionicity of twin pregnancy
\end{abstract}

Ginekologia Polska 2022; 93, 4: 310-313

\section{INTRODUCTION}

Owing to a high rate of complications, twin pregnancies are regarded as pathological in humans. In Europe, they are related to nine-fold higher risk of preterm birth, twelve-fold higher risk of very preterm birth and seven-fold higher risk of neonatal death in comparison to singletons [1]. Moreover, incidence of twin pregnancies has grown dramatically over the last several decades as a result of the increase of the maternal age at conception and introduction of assisted reproduction techniques $[2,3]$. In 2010, the twinning rate in Europe ranged from 1:110 in Romania up to 1:38 in Cyprus [1], which makes a twin pregnancy a very common challenge of contemporary prenatal medicine.
General classification of twin pregnancies is based on the number of zygotes from which the twin foeti develop (dizygotic/DZ or monozygotic/MZ), the number of placental masses (dichorionic/DC or monochorionic/MC) and the number of amniotic sacs (diamniotic/DA or monoamniotic/MA) [4]. DZ pregnancies are almost always DC DA with each foetus having its own placenta and amniotic cavity. On the other hand, chorionicity and amniocity of MZ pregnancies is determined by the time at which division of an embryo occurs [5]. Thus, fertilisation of one ovum by one sperm leading to $M Z$ twin pregnancy may result in DC DA (division within 4 days of conception), MC DA (division between 4 and 8 days after conception) or MC MA gestation (division between 8 and 12 days after conception) [4].

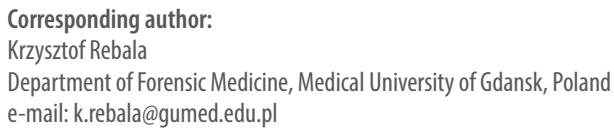


Chorionicity of twin pregnancies is diagnosed routinely by high-resolution ultrasound examination based on the number of placental masses, intertwin membrane thickness and the presence or absence of lambda or T sign between 10 and 14 weeks [6]. It is assumed that knowledge of chorionicity is paramount when managing a twin pregnancy [7] as far as MC pregnancies have 3-5 times higher perinatal morbidity and mortality in comparison to DC pregnancies [8]. However, as far as MC pregnancies develop only in case of MZ twin foeti, a prognostic value for the course of a twin pregnancy may be provided also by a DNA test determining pregnancy zygosity, especially when chorionicity cannot be reliably determined [9]. Mortality of foeti is at least 2.5 times higher in $\mathrm{MZ}$ than in DZ twin pregnancies [10,11], and this is mainly due to complications in MC pregnancies, which constitute roughly two-thirds of MZ twin pregnancies [12]. Recently, several techniques have been developed for non-invasive prenatal twin zygosity testing of cell-free foetal DNA circulating in maternal plasma, based on sequencing of single nucleotide polymorphisms [13-15], sequencing of microhaplotypes [16] and genotyping of multiallelic microsatellites [9].

\section{Objectives}

The aim of our study was to evaluate and compare the potential of DNA analysis and ultrasound examination for diagnosis of high-risk and low-risk twin pregnancies. For the purpose of our study, zygosity of 42 twin pregnancies was determined immediately after the birth and confronted with results of routine ultrasound examination of pregnancy chorionicity in the first trimester of gestation.

\section{MATERIAL AND METHODS}

A total of 42 women with twin pregnancies were involved in the study. They were patients of the Department of Obstetrics at the Medical University of Gdańsk and of the Department of Obstetrics and Gynaecology at the St. Adalbert Hospital in Gdańsk, Copernicus Podmiot Leczniczy Sp. z o.o. The study was approved by the Independent Bioethics Commission for Research at the Medical University of Gdańsk, and all patients provided their informed written consent for participation in the study. Chorionicity was determined by an attending physician dedicated to obstetric ultrasound during routine high-resolution sonographic examination between 10 and 14 weeks of gestation, based on the number of placental masses, the intertwin membrane thickness and the presence or absence of lambda or T sign [6]. Immediately after delivery, about $1.5 \mathrm{~mL}$ of umbilical cord blood was collected from each twin (84 samples in total) from the side of a placenta after cutting an umbilical cord. DNA was isolated from the umbilical cord blood samples with the use of a non-enzymatic method [17]. DNA concentration was measured spectrophotometrically with the use of an ND-
1000 spectrophotometer (NanoDropTechnologies, Rockland, $\mathrm{DE}$, USA). A total of 22 autosomal short tandem repeats (STRs), one Y-chromosomal STR and a gender marker (amelogenin) were amplified with the use of a commercial PowerPlex Fusion kit (Promega, Madison, WI, USA) and a Mastercycler Gradient thermal cycler (Eppendorf, Hamburg, Germany) in a total volume of $5 \mu \mathrm{L}$, containing $1 \mathrm{ng}$ of DNA. Amplification products were suspended in Super-DI Formamide (MCLAB, South San Francisco, CA, USA), mixed with WEN ILS 500 size standard (Promega), denatured and analysed by capillary electrophoresis in a 3130 Genetic Analyzer (Applied Biosystems, Foster City, CA, USA) with the use of NanoPOP-4 polymer (MCLAB) and a 36-cm 4-capillary array. Genotypes of the analyzed samples were determined with the use of GeneMapper ID software v3.2 (Applied Biosystems) by comparison to an allelic ladder provided with the PowerPlex Fusion kit.

\section{RESULTS}

Routine ultrasound imaging in the first trimester of twin gestations revealed 21 DC DA (50.0\%) and 21 MC pregnancies (50.0\%). Among $21 \mathrm{MC}$ pregnancies, there were $17 \mathrm{DA}$ pregnancies (81.0\%), 2 MA pregnancies (9.5\%) and 2 pregnancies with unknown amniocity.

Human identity testing by STR typing of samples of the umbilical cord blood from twins coming from 42 pregnancies showed 23 twin pairs with different genotypes (DZ pregnancies, 54.8\%) and 19 twin pairs with identical genotypes (MZ pregnancies, 45.2\%; Tab. 1). Among $19 \mathrm{MZ}$ pregnancies, there were 2 DC DA pregnancies (10.5\%) and $17 \mathrm{MC}$ pregnancies (89.5\%), including $13 \mathrm{MCDA}$ pregnancies (68.4\%), 2 MC MA pregnancies (10.5\%) and 2 MC pregnancies with unknown amniocity. On the other hand, among $23 \mathrm{DZ}$ pregnancies, 19 pregnancies were diagnosed by ultrasound examination as DC DA (82.6\%). Remaining 4 DZ pregnancies were identified by physicians dedicated to obstetric ultrasound as MC DA (17.4\%) and constituted $19.0 \%$ of all high-risk MC pregnancies detected by ultrasound imaging.

\section{DISCUSSION}

Chorionicity is the main determinant of the perinatal outcome in twin pregnancies as far as perinatal morbidity and mortality are much higher in MC than DC pregnancies. This is mainly due to complications associated with monochorionicity, resulting from development of intertwin vascular anastomoses in the shared placenta and including twin-to-twin transfusion syndrome, twin reversed arterial perfusion sequence and selective intrauterine growth restriction. Therefore, the accurate prenatal diagnosis of chorionicity is of major clinical importance in the management of twin pregnancies as far as it allows provision of increased surveillance in MC pregnancies and detection of complications in their early stages [7]. Ultrasound exami- 
Table 1. Classification of $\mathbf{4 2}$ twin pregnancies by zygosity, chorionicity and amniocity, as revealed by postnatal short tandem repeat typing and routine ultrasound examination in the first trimester of gestation

\begin{tabular}{|l|l|l|l|l|l|}
\hline Zygosity & Chorionicity & & Amniocity & \\
\hline Dizygotic & $23(55 \%)$ & Dichorionic & $19(45 \%)$ & Diamniotic & $19(45 \%)$ \\
\hline & & Monochorionic & $4(10 \%)$ & Diamniotic & $4(10 \%)$ \\
\hline Monozygotic & $19(45 \%)$ & Dichorionic & $2(5 \%)$ & Diamniotic & $2(5 \%)$ \\
\hline & & Monochorionic & $17(40 \%)$ & Diamniotic & $13(31 \%)$ \\
\hline & & & Monoamniotic & $2(5 \%)$ \\
\hline
\end{tabular}

nation performed every two weeks of MC pregnancy is more likely to result in early diagnosis of twin-to-twin transfusion syndrome in comparison to traditional monthly assessment, regarded as sufficient for management of DC pregnancies [4]. Moreover, in order to minimise perinatal deaths, delivery should be considered earlier in MC than DC pregnancies (36 vs 37 weeks of gestation) [18].

However, ascertainment of twin pregnancy chorionicity by ultrasound examination may be problematic when a pregnant woman misses her early gestation scan, when she is obese or when amniotic fluid volume is reduced [19]. Furthermore, there are cases of MC pregnancies with a visualised lambda sign between 10 and 14 weeks of gestation, thus incorrectly diagnosed as low-risk DC pregnancies [20]. Reverse misdiagnoses of DC as MC pregnancies prior to 14 weeks of gestation are also encountered in medical practice [21]. In our study, as much as $10 \%$ of pregnancies (4 cases) were misdiagnosed during the early ultrasound scan as high-risk MC gestations, but were found to be DZ by DNA testing, which may be explained as a result of fusion of DZ placentas [22]. These four misdiagnoses were made at two different obstetric units in Gdańsk by different physicians dedicated to obstetric ultrasound and with the use of different equipment, which excludes systematic error of one examiner as explanation of the observed discrepancies. It should be noted that dizygosity detected prenatally eliminates the need for precise ultrasound chorionicity assessment and frequent prenatal visits as far as DZ twins are DC even in case of fused placentas which generally do not form anastomoses resulting in twin-to-twin transfusion syndrome, as observed in MC pregnancies [23]. On the other hand, if chorionicity cannot be unequivocally determined, prenatal diagnosis of $\mathrm{MZ}$ pregnancy does not solve the problem of planned care for a pregnant woman. In such cases, a more pessimistic variant of MC pregnancy should always be assumed as far as it is safer to examine a pregnant woman unnecessarily every two weeks than to detect complications too late.

If evaluation of chorionicity is unclear, approximately $50 \%$ of DZ pregnancies may be identified as low-risk DC pregnan- cies by ultrasound examination in the mid-pregnancy, based on opposite sex of twins. Although determination of sex of foeti is possible also in the first trimester, it is characterised by high risk of incorrect assignments [24]. However, a number of cases of postzygotic genome alterations in MC pregnancies, resulting in twin foeti of opposite sex, have been described [25-27]. Two separate placental masses are also indicative of low-risk DC pregnancy, but they are seen only in about one-third of twin pregnancies [4]. On the other hand, in European populations, as much as two-thirds of twin pregnancies are DZ $[28,29]$. Thus, a sensitive and specific DNA test determining pregnancy zygosity, carried out alone in the early or middle pregnancy, restricts high-risk twin gestation diagnosis to $33 \%$ of twin pregnancies which are MZ. Although sonographic evaluation of chorionicity distinguishes more accurately high-risk and low-risk twin pregnancies, limiting high-risk twin gestation diagnosis to $25 \%$ of twin pregnancies which are MC [4], we have identified in our study as much as $10 \%$ of cases misdiagnosed sonographically as high-risk MC pregnancies, which could have been managed from the very beginning as low-risk pregnancies if a prenatal DNA test of zygosity had been available and performed.

A certain limitation of our study is the fact that zygosity of twins was evaluated postnatally, whereas currently available DNA tests for prenatal zygosity testing may be characterised by lower sensitivity of correct diagnosis of zygosity and by higher DNA input requirements [9, 13, 14]. However, the aim of our study was not to assess robustness and accuracy of a chosen DNA-based prenatal zygosity testing technique, but to evaluate general potential of DNA zygosity tests for non-invasive evaluation of risk of complications in twin pregnancies. Our results demonstrate that highly sensitive and specific DNA tests for prenatal diagnostics of twin pregnancy zygosity are needed and should be further developed.

\section{CONCLUSIONS}

Our study based on postnatal twin pregnancy zygosity testing, confronted with results of ultrasound examination in the first trimester, indicates a high potential of prenatal DNA 
testing of zygosity in identification of low-risk and high-risk twin gestations requiring different prenatal care in clinical practice, especially in cases when chorionicity and zygosity cannot be reliably determined by ultrasound examination and as a supplementary test able to detect gestations misdiagnosed as MC, resulting from fusions of DZ placentas. In such cases, dizygosity detected prenatally eliminates the need for frequent prenatal visits typical for MC pregnancies. If chorionicity cannot be unequivocally determined and a prenatal DNA test detects $M Z$ twins, a more pessimistic variant of MC pregnancy should always be assumed.

\section{Funding}

Financed by the statutory activity subsity for the Medical University od Gdansk.

\section{Conflict of interest}

No potential conflicts of interest were reported by the authors.

\section{REFERENCES}

1. Heino A, Gissler M, Hindori-Mohangoo AD, et al. Euro-Peristat Scientific Committee. Variations in Multiple Birth Rates and Impact on Perinatal Outcomes in Europe. PLoS One. 2016; 11(3): e0149252, doi: 10.1371/journal.pone.0149252, indexed in Pubmed: 26930069.

2. Pison G, D'Addato AV. Frequency of twin births in developed countries. Twin Res Hum Genet. 2006; 9(2): 250-259, doi: 10.1375/183242706776382338, indexed in Pubmed: 16611495.

3. Pison G, Monden C, Smits J. Twinning Rates in Developed Countries: Trends and Explanations. Population and Development Review. 2015; 41(4): 629-649, doi: 10.1111/j.1728-4457.2015.00088.x.

4. Moise KJ, Argoti PS. Society for Maternal-Fetal Medicine. The importance of determining chorionicity in twin gestations. Contemp Ob Gyn. 2013; 58: $35-43$.

5. Fuchs K, D'Alton M. Dichorionic Diamniotic Twin Gestations. Obstetric Imaging: Fetal Diagnosis and Care. 2018:648-650.e1, doi: 10.1016/b9780-323-44548-1.00161-3.

6. Sepulveda W, Sebire NJ, Hughes K, et al. The lambda sign at 10-14 weeks of gestation as a predictor of chorionicity in twin pregnancies. Ultrasound Obstet Gynecol. 1996; 7(6): 421-423, doi: 10.1046/j.1469-0705 1996.07060421.x, indexed in Pubmed: 8807758.

7. D'Addario V, Rossi C. Diagnosis of chorionicity: The role of ultrasound. Diagnóstico Prenatal. 2014; 25(2): 58-64, doi: 10.1016/j.diapre.2013.09.004.

8. Bajoria R, Kingdom J. The case for routine determination of chorionicity and zygosity in multiple pregnancy. Prenat Diagn. 1997; 17(13): 1207-1225, indexed in Pubmed: 9509540.

9. Dziennik A, Preis K, Świątkowska-Freund M, et al. Genotyping of STR and DIP-STR Markers in Plasma Cell-Free DNA for Simple and Rapid Noninvasive Prenatal Diagnosis of Zygosity of Twin Pregnancies. Twin Res Hum Genet. 2019; 22(5): 321-329, doi: 10.1017/thg.2019.89, indexed in Pubmed: 31619303.

10. Monteagudo A, Timor-Tritsch I, Sharma S. Early and simple determination of chorionic and amniotic type in multifetal gestations in the first fourteen weeks by high-frequency transvaginal ultrasonography. American Journal of Obstetrics and Gynecology. 1994; 170(3): 824-829, doi: 10.1016/s0002-9378(94)70291-8.

11. Landy HJ, Nies BM. The vanishing twin. In: Keith LG, Papiernik E, Keith DM. ed. Multiple Pregnancy: Epidemiology, Gestation and Perinatal Outcome, 1st ed. The Parthenon Publishing Group, New York 1995: 59-71.

12. Simpson L, Miller R. Twin-Twin Transfusion Syndrome. Obstetric Imaging: Fetal Diagnosis and Care. 2018: 651-659.e2, doi: 10.1016/b978-0-32344548-1.00162-5.
13. Qu JZZ, Leung TY, Jiang $P$, et al. Noninvasive prenatal determination of twin zygosity by maternal plasma DNA analysis. Clin Chem. 2013; 59(2): 427-435, doi: 10.1373/clinchem.2012.194068, indexed in Pubmed: 23115054.

14. Zheng J, Xu C, Guo J, et al. Effective noninvasive zygosity determination by maternal plasma target region sequencing. PLoS One. 2013; 8(6): e65050, doi: 10.1371/journal.pone.0065050, indexed in Pubmed: 23762285.

15. Norwitz ER, McNeill G, Kalyan A, et al. Validation of a Single-Nucleotide Polymorphism-Based Non-Invasive Prenatal Test in Twin Gestations: Determination of Zygosity, Individual Fetal Sex, and Fetal Aneuploidy. J Clin Med. 2019; 8(7), doi: 10.3390/jcm8070937, indexed in Pubmed: 31261782.

16. Bai Z, Zhao Hu, Lin S, et al. Evaluation of a Microhaplotype-Based Noninvasive Prenatal Test in Twin Gestations: Determination of Paternity, Zygosity, and Fetal Fraction. Genes (Basel). 2020; 12(1), doi: 10.3390/genes12010026, indexed in Pubmed: 33375453.

17. Lahiri DK, Nurnberger JI. A rapid non-enzymatic method for the preparation of HMW DNA from blood for RFLP studies. Nucleic Acids Res. 1991; 19(19): 5444, doi: 10.1093/nar/19.19.5444, indexed in Pubmed: 1681511.

18. Cheong-See F, Schuit E, Arroyo-Manzano D, et al. Global Obstetrics Network (GONet) Collaboration. Prospective risk of stillbirth and neonatal complications in twin pregnancies: systematic review and meta-analysis. BMJ. 2016; 354: i4353, doi: 10.1136/bmj.i4353, indexed in Pubmed: 27599496.

19. Lee KA, Oh KJ, Lee SMi, et al. The frequency and clinical significance of twin gestations according to zygosity and chorionicity. Twin Res Hum Genet. 2010; 13(6): 609-619, doi: 10.1375/twin.13.6.609, indexed in Pubmed: 21142938.

20. Yamashita A, Ishii K, Hidaka N, et al. Monochorionic monozygotic twin pregnancy complicated with twin-twin transfusion syndrome presenting with an obvious lambda sign in the first trimester. Fetal Diagn Ther. 2015; 37(2): 154-156, doi: 10.1159/000367969, indexed in Pubmed: 25633149.

21. Stenhouse $E$, Hardwick C, Maharaj S, et al. Chorionicity determination in twin pregnancies: how accurate are we? Ultrasound Obstet Gynecol. 2002; 19(4): 350-352, doi: 10.1046/j.1469-0705.2002.00679.x, indexed in Pubmed: 11952963.

22. Calomfirescu-Avramescu A, Demetrian M, Grecu G, et al. Placenta fusion in a dichorionic-diamniotic IVF twin pregnancy - case presentation. Romanian Journal of Medical Practice. 2020; 15(1): 97-100, doi: 10.37897/rjmp.2020.1.18.

23. Benirschke K. The biology of the twinning process: how placentation influences outcome. Semin Perinatol. 1995; 19(5): 342-350, doi: 10.1016/s0146-0005(05)80012-6, indexed in Pubmed: 8821022.

24. Efrat Z, Akinfenwa OO, Nicolaides KH. First-trimester determination of fetal gender by ultrasound. Ultrasound Obstet Gynecol. 1999; 13(5): 305-307, doi: 10.1046/j.1469-0705.1999.13050305.x, indexed in Pubmed: 10380292

25. Wachtel SS, Somkuti SG, Schinfeld JS. Monozygotic twins of opposite sex. Cytogenet Cell Genet. 2000; 91(1-4): 293-295, doi: 10.1159/000056859, indexed in Pubmed: 11173871

26. Zech NH, Wisser J, Natalucci G, et al. Monochorionic-diamniotic twins discordant in gender from a naturally conceived pregnancy through postzygotic sex chromosome loss in a $47, X X Y$ zygote. Prenat Diagn. 2008; 28(8): 759-763, doi: 10.1002/pd.2031, indexed in Pubmed: 18567067.

27. Bohec C, Douet-Guilbert N, Basinko A, et al. Difficult diagnosis and management of an heterokaryotypic monochorionic twin pregnancy with discordant fetal sex and 45,X/47,XYY karyotypes. Fetal Pediatr Pathol. 2010; 29(6): 424-430, doi: 10.3109/15513815.2010.505630, indexed in Pubmed: 21043568.

28. Machin GA. Conjoined twins: implications for blastogenesis. Birth Defects Orig Artic Ser. 1993; 29(1): 141-179, indexed in Pubmed: 8280870 .

29. Blickstein I, Verhoeven H, Keith L. Zygotic Splitting after Assisted Reproduction. N Engl J Med. 1999; 340(9): 738-739, doi: 10.1056/nejm199903043400916. 